\title{
B7-H4 expression in human infiltrating ductal carcinoma-associated macrophages
}

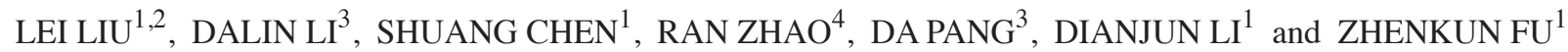 \\ ${ }^{1}$ Department of Immunology, Heilongjiang Provincial Key Laboratory for Infection and Immunity, \\ Heilongjiang Academy of Medical Sciences; ${ }^{2}$ College of Bioinformatics Science and Technology, \\ Harbin Medical University; ${ }^{3}$ Department of Breast Surgery, The Third Affiliated Hospital of Harbin Medical University; \\ ${ }^{4}$ Department of Pathology, Harbin Medical University, Harbin, Heilongjiang 150081, P.R. China
}

Received June 30, 2015; Accepted July 6, 2016

DOI: $10.3892 / \mathrm{mmr} .2016 .5510$

\begin{abstract}
B7-H4 is a co-inhibitory molecule of the B7 family, which is expressed on antigen-presenting cells (APCs) and is able to limit the T-cell immune response. Macrophages act as professional APCs and are important for immunoregulation of the tumor microenvironment in breast cancer. In order to identify the association between the presence of B7-H4 on macrophages and infiltrating ductal carcinoma (IDC), the present study investigated the expression of B7-H4 on macrophages with different polarizations. The expression levels of B7-H4 in IDC tissues were determined using immunohistochemistry, and the expression of $\mathrm{B} 7-\mathrm{H} 4$ on macrophages in the breast IDC microenvironment were determined using western blot analysis and reverse transcription-quantitative polymerase chain reaction (RT-qPCR). The expression levels of interleukin (IL)-6 and IL-10 were detected in IDC tissues and the supernatants of polarized macrophages using an enzyme-linked immunosorbent assay and RT-qPCR. The present study demonstrated that B7-H4 was overexpressed in IDC tissues and macrophages. In vitro, M1 and M2 macrophages exhibited different expression levels of B7-H4. IL-6 and -10 exhibited
\end{abstract}

Correspondence to: Professor Zhenkun Fu or Professor Dianjun Li, Department of Immunology, Heilongjiang Provincial Key Laboratory for Infection and Immunity, Heilongjiang Academy of Medical Sciences, Harbin Medical University, 157 Baojian Street, Harbin, Heilongjiang 150081, P.R. China

E-mail: zhenkun_fu@aliyun.com

E-mail: lidj1945@126.com

Abbreviations: IDC, infiltrating ductal carcinoma; ER, estrogen receptor; PR, progesterone receptor; HER2, human epidermal growth factor receptor-2; AJCC, American Joint Committee on Cancer; IL, interleukin; IFN- $\gamma$, interferon- $\gamma$; GM-CSF, granulocyte-macrophage colony-stimulating factor; M-CSF, macrophage colony-stimulating factor; PBMC, peripheral blood monouclear cells; LPS, lipopolysaccharides; IHC, immunohistochemistry

Key words: B7-H4, macrophage, infiltrating ductal carcinoma, interleukin-6, interleukin-10 higher expression in the IDC tissues compared with in distal pericarcinomatous tissues. In conclusion, B7-H4 exhibited overexpression in IDC tissues and cultured macrophage cells. Furthermore, M2 macrophages exhibited higher expression levels of B7-H4 compared with the M1 subtype. In addition, IL-6 and -10 may be associated with B7-H4 expression on macrophages of different polarizations in the IDC microenvironment.

\section{Introduction}

Infiltrating ductal carcinoma (IDC) is one of the most common malignant tumors in female breast cancer (1). Tumor-specific immune promoting and inhibiting responses are important for the pathogenesis of IDC (2). CD4+ regulatory T cells at tumor sites may significantly suppress immune responses, leading to immune tolerance of breast cancer cells (3). Conversely, $\mathrm{CD} 8^{+} \mathrm{T}$ cells may lead to an antitumor response against neoplastic cells (4). A recent study suggested that the plasticity of tumor-infiltrated T-cell subsets was influenced by several factors, including the tumor microenvironment and antigen-presenting cells (APCs) (5). APCs, such as macrophages, may regulate the differentiation of $\mathrm{T}$ cells via co-stimulatory/inhibitory molecules expressed on the surface of cells and soluble products, including various cytokines, such as interleukin (IL)-10 and -6. Based on the polarization of type 1 and type $2 \mathrm{~T}$ helper cells (Th1 and Th2), macrophages may be divided into the classically activated (M1) macrophage phenotype and the alternatively activated (M2) macrophage phenotype (6,7). M1 macrophages promote the antitumor response of T cells, and M2 macrophages promote regulatory immune responses and enhance tumor growth. However, the exact function of tumor-infiltrated macrophages in the progression of IDC remains to be elucidated (8-10).

B7 co-stimulatory/inhibitory family molecules are expressed by macrophages and tumor cells, and are important regulators of the balance between antitumor and tumor-promoting immune responses. CD80/86 promotes interferon- $\gamma($ IFN- $\gamma)$ expression, which in turn mediates the Th1 response, and are frequently expressed by M1 macrophages. Programmed death-ligand 1 (PD-L1)/L2 and B7-H4 reduce T-cell responses and are expressed by M2 macrophages. 
B7-H4, also termed B7x and B7S1, is a co-inhibitory molecule for T-cell activation signaling. Due to this function, B7-H4 may limit the proliferation, cytokine secretion and the development of cytotoxicity of $\mathrm{T}$ cells, including $\mathrm{CD}^{+}$and $\mathrm{CD}^{+}$ $\mathrm{T}$ cells $(11,12)$. The expression of the co-inhibitory molecule B7-H4 in cancer cells may be associated with tumor progression, due to its importance in the tumor microenvironment and its significance in the activation of $\mathrm{T}$ cells. The present study demonstrated that B7-H4 may be overexpressed in the breast IDC microenvironment, and tumor-infiltrated macrophages may also express B7-H4. However, the factor that induces B7-H4 expression in macrophages remains to be elucidated. It is possible that specific cytokines in the breast IDC microenvironment may be associated with $\mathrm{B} 7-\mathrm{H} 4$ expression in macrophages.

The present study characterized B7-H4 expression in tumor-infiltrated macrophages in situ. In addition, in vitro experiments revealed that macrophages of different polarizations may express various levels of B7-H4. In congruence with this, the M1 distinctive cytokine IL-6 and the M2 distinctive cytokine IL-10 (13) were increased in the IDC microenvironment when compared with pericarcinomatous (PC) tissue. Furthermore, different expression levels of IL-6 and -10 were detected in M1 and M2 phenotype cell cultures. The present study revealed the association between B7-H4 and specialized subpopulations of macrophages, along with the potential influence B7-H4 expression may have in the IDC microenvironment.

\section{Materials and methods}

Human tissue biopsies. Paired IDC and PC tissues were collected from 61 patients with IDC were obtained from Outdo BioTech Co., Ltd. (Shanghai, China). In addition, six frozen IDC samples from breast cancer surgery between 2008 and 2012 were obtained from the tissue bank in the Third Affiliated Hospital of Harbin Medical University (Harbin, China) for immunofluorescence. The patients had a clear pathological diagnosis according to the American Joint Committee on Cancer (AJCC) staging system. The present study was approved by the ethics committee of the Third Affiliated Hospital of Harbin Medical University and all patients provided written informed consent. In addition, the expression levels of estrogen receptor (ER), progesterone receptor (PR) and human epidermal growth factor receptor 2 (HER2) were detected in 31 patients. For paraffin-embedded sections of IDC and PC samples, the reagents for detection of ER, PR and HER2 were obtained from Maixin Biotech Co., Ltd (Fuzhou, China).

Cell culture, and M1 and M2 phenotype macrophage polarization. Human blood monocytes were obtained from peripheral blood mononuclear cells (PBMCs), which were obtained from healthy female volunteer blood samples (mean age, 34.5 years), using Miltenyi Biotec MACS Separator Starting kits and Human CD14 MicroBeads (Miltenyi Biotec Ltd., Surrey, UK).

M0 cells are human blood monocytes without cytokine stimulus, they were used as control cells in the present study. The M1 phenotype of cells may be induced by granulocyte-macrophage colony-stimulating factor (GM-CSF), IFN- $\gamma$ and lipopolysaccharides (LPS) treatments. The M2 phenotype of cells may be induced by M-CSF, IL-4 and IL-13. All of the cytokines used were obtained from PeproTech, Inc. (Rocky Hill, NJ, USA). GM-CSF and M-CSF were added to the blood monocytes in RPMI 1640 (GE Healthcare Life Sciences, Little Chalfont, UK) supplemented with $10 \%$ fetal bovine serum (Biological Industries, Beit Haemek, Israel). After a $48 \mathrm{~h}$ incubation at $37^{\circ} \mathrm{C}, \mathrm{IFN}-\gamma$ and LPS were added to the M1 phenotype culture media, and IL-4 and IL-13 were added to the M2 culture media (13). Following a further $48 \mathrm{~h}$ incubation, the M1 and M2 cells underwent the following experiments. The concentrations of GM-CSF and M-CSF used were $30 \mathrm{ng} / \mathrm{ml}$, the remaining cytokines were used at $10 \mathrm{ng} / \mathrm{ml}$. Similar manipulations to obtain the M1 and M2 phenotypes were performed on the Thp1 human monocyte cell line (Bioleaf Biotech, Co., Ltd., Shanghai, China).

Enzyme-linked immunosorbent assay (ELISA). The supernatant of the tissue samples was acquired following incubation with a lysis buffer (from ELISA kits) and ultrasonic processing. The lysates were analyzed using RayBio Human IL-6 and IL-10 ELISA kits (RayBio, Inc., Norcross, GA, USA) according to the manufacturer's protocol. In addition, the supernatant of the cultured monocytes, M1 and M2 phenotypes, was collected and were analyzed using RayBio Human IL-6 and IL-10 ELISA kits.

Immunohistochemistry (IHC) and scoring. The paraffin-embedded breast IDC and PC tissue samples were prepared by Outdo BioTech Co., Ltd., they were sectioned (4 $\mu \mathrm{m})$ using a Leica RM2245 microtome (Leica Microsystems, Wetzlar, Germany). The samples were stained with rabbit anti-B7-H4 monoclonal primary immunoglobulin (Ig)G antibody (1:300, GeneTex, Inc., Irvine, CA, USA; cat. no. GTX42699) overnight at $4^{\circ} \mathrm{C}$ with agitation, followed by ImmunoCruz rabbit LSAB Staining system (Santa Cruz Biotechnology, Inc., Dallas, TX, USA; cat. no. sc-2051). Subsequently, images were obtained using Nikon Eclipse 80i microscope (Nikon Corporation, Tokyo, Japan).

IHC scoring was performed on the 61 paired IDC and PC samples from patients with IDC (14). Proportion and intensity scores were calculated for each sample. The intensity of immunostaining was scored by visual assessment of the intensity (brown color) in positively stained cells: 0 , none; 1 , weak; 2 , intermediate and 3 , strong. The proportion score represented the percentage of positively stained cells in the entire tissue section: 0 , none; $1,<5 \% ; 2,5-25 \% ; 3,26-50 \%$; $4,51-75 \%$ and $5,>75 \%$. Overall B7-H4 expression in the IDC and PC samples was expressed as a histoscore, which was the sum of the proportion (0-5) and the intensity scores (0-3), producing a range between $0-8$, with a maximum possible score of 8 .

Immunofluorescence. Frozen breast IDC and PC tissue samples were sectioned using a Microm HM525 freezing microtome (Thermo Fisher Scientific, Inc., Waltham, MA, USA). The specimens were stained with primary antibodies overnight at $4^{\circ} \mathrm{C}$, as follows: Rabbit anti-B7-H4 polyclonal 
primary IgG antibody (1:200, Santa Cruz Biotechnology, Inc.; cat. no. 68872), mouse anti-CD68 monoclonal IgG antibody (1:200, Santa Cruz Biotechnology, Inc.; cat. no. 20060), rabbit anti-CD163 polyclonal primary IgG antibody (1:200; Abcam, Cambridge, UK; cat. no. ab87099), followed by Alexa Fluor 488-conjugated donkey anti-mouse IgG and Alexa Fluor 555-conjugated donkey anti-rabbit IgG (1:200; Abcam; cat. no. ab150105) for $1 \mathrm{~h}$ at $37^{\circ} \mathrm{C}$ in the dark. The DAPI solution $(1 \mu \mathrm{g} / \mathrm{ml}$; Solarbio Science \& Technology Co., Ltd., Beijing, China) was used for nuclear staining. The images were obtained using a Nikon Eclipse 80i fluorescence microscope.

Western blot analysis. The macrophages obtained from PBMCs and Thpl cells were resuspended in lysis buffer and $2 \mathrm{mM}$ phenylmethane sulfonyl fluoride (Solarbio Science \& Technology Co., Ltd.). Lysates were centrifuged at 12,000 x g for $5 \mathrm{~min}$ at $4^{\circ} \mathrm{C}$. The protein concentration was quantified by BCA Protein Assay kit (Solarbio Science \& Technology Co., Ltd.) and $40 \mu \mathrm{g}$ was loaded into $12 \%$ SDS-PAGE gels. The proteins were transferred to nitrocellulose membranes. Subsequently, the expression levels of B7-H4 were detected by immunoblotting using $\beta$-actin as a housekeeping protein. The membranes were blocked with $5 \%$ skim milk at room temperature for $1 \mathrm{~h}$. The primary antibodies used were polyclonal rabbit anti-B7-H4 IgG antibody (1:300; Santa Cruz Biotechnology, Inc.; cat. no. sc-68872) and mouse anti- $\beta$-actin monoclonal antibody (1:4,000; ProteinTech Group, Inc., Chicago, IL, USA; cat. no. 66009-1) at $4^{\circ} \mathrm{C}$ overnight. The secondary antibodies used were horseradish peroxidase (HRP)-conjugated affinipure goat anti-rabbit (1:2,000; cat. no. SA00001-1) and anti-mouse IgG (1:2,000; ProteinTech Group, Inc.; cat. no. SA00001-2) at $37^{\circ} \mathrm{C}$ for $1 \mathrm{~h}$. The Luminata Forte Western HRP substrate (EMD Millipore, Billerica, MA, USA) was used for enhanced chemiluminescence visualization. Finally, the rabbit anti-human B7-H4 monoclonal primary IgG antibody (1:300, GeneTex, Inc.) was used as a repeated verification test. The western blots were analyzed sing ImageJ 1.48 software (imagej.nih.gov).

Reverse transcription-quantitative polymerase chain reaction $(R T-q P C R)$. RNA was extracted from macrophages obtained from PBMCs and Thpl cells using TRIzol ${ }^{\circledR}$ reagent (Invitrogen; Thermo Fisher Scientific., Inc.). High-Capacity cDNA Reverse Transcription kit (Thermo Fisher Scientific, Inc.) was used for mRNA RT. The RT was conducted as follows: $25^{\circ} \mathrm{C}$ for $10 \mathrm{~min} ; 37^{\circ} \mathrm{C}$ for $2 \mathrm{~h} ; 85^{\circ} \mathrm{C}$ for $5 \mathrm{~min}$; and then paused at $12^{\circ} \mathrm{C}$. The primer sequences used for RT-qPCR were as follows: B7-H4, forward (F) 5'-TCTGGGCATCCC AAGTTGAC-3', and reverse (R) 5'-TCCGCCTTTTGATCT CCGATT-3'; IL-6, F 5'-ACTCACCTCTTCAGAACGAAT TG-3' and R 5'-CCATCTTTGGAAGGTTCAGGTTG-3'; and IL-10, F 5'-GACTTTAAGGGTTACCTGGGTTG-3' and R 5'-TCACATGCGCCTTGATGTCTG-3'. The primer sequences for the control, glyceraldehyde 3-phosphate dehydrogenase, were as follows: F 5'-CAAGTTCAACGGCAC AGTCAA-3' and R 5'-GTGGTCATGAGCCCTTCCA-3'. The primers were obtained from Invitrogen (Thermo Fisher Scientific, Inc.). The reaction was performed using ABI Power SYBR Green PCR Master mix on a ABI StepOne
Table I. Clinical characteristics of patients with IDC and the association between B7-H4 expression in IDC and PC tissues.

\begin{tabular}{|c|c|c|c|}
\hline Variable & IDC & $\mathrm{PC}$ & P-value \\
\hline \multicolumn{4}{|l|}{ Clinical staging ${ }^{\mathrm{a}}$} \\
\hline I & 5 & $\mathrm{~N} / \mathrm{A}$ & N/A \\
\hline II & 31 & $\mathrm{~N} / \mathrm{A}$ & N/A \\
\hline III & 14 & $\mathrm{~N} / \mathrm{A}$ & N/A \\
\hline \multicolumn{4}{|l|}{ ER } \\
\hline+ & 19 & $\mathrm{~N} / \mathrm{A}$ & N/A \\
\hline- & 12 & N/A & N/A \\
\hline \multicolumn{4}{|l|}{ PR } \\
\hline+ & 15 & $\mathrm{~N} / \mathrm{A}$ & N/A \\
\hline- & 16 & N/A & N/A \\
\hline \multicolumn{4}{|l|}{ HER2 } \\
\hline+ & 12 & $\mathrm{~N} / \mathrm{A}$ & N/A \\
\hline- & 19 & N/A & N/A \\
\hline Age (years) & $53.28 \pm 7.79$ & $\mathrm{~N} / \mathrm{A}$ & N/A \\
\hline B7-H4 expression ${ }^{b}$ & $7.10 \pm 0.78$ & $4.75 \pm 2.08$ & $0.011^{\mathrm{c}}$ \\
\hline
\end{tabular}

IDC tissues, $n=61$. Clinical staging, $n=50$. ER, PR and HER2 expression, $\mathrm{n}=31$. The mean IHC scores \pm standard error of the mean were calculated for B7-H4 expression in IDC and PC tissues. ${ }^{\mathrm{a}} \mathrm{Ac}-$ cording to the American Joint Committee on Cancer. ${ }^{\mathrm{b}} \mathrm{IHC}$ scoring. ${ }^{\mathrm{C}} \mathrm{P}<0.05 \mathrm{PC}$ vs. IDC tissues. IDC, infiltrating ductal carcinoma; PC, pericarcinomatous; +, positive; -, negative; ER, estrogen receptor; $\mathrm{PR}$, progesterone receptor; HER2, human epidermal growth factor receptor-2; IHC, immunohistochemistry.

Real-Time PCR system (Applied Biosystems; Thermo Fisher Scientific, Inc.). The thermocycling conditions were as follows: $95^{\circ} \mathrm{C}$ for $10 \mathrm{~min}$; 40 cycles of $95^{\circ} \mathrm{C}$ for $20 \mathrm{sec}$ and $60^{\circ} \mathrm{C}$ for $30 \mathrm{sec}$. The results were quantified and analyzed using a $\mathrm{Cq}$ method $(15,16)$ and melting curve analysis.

Flow cytometry. The present study determined the purity of monocytes from PBMCs using mouse fluorescein isothiocyanate-conjugated anti-IgG CD14 (1 $\mu \mathrm{g} / \mathrm{test}$; cat. no. 11-0141-81). The M1 phenotype was verified using mouse phycoerythrin-conjugated anti-CD86 IgG $(0.5 \mu \mathrm{g} /$ test; cat. no. 12-0861-81) and M2 cells were verified by mouse APC-conjugated anti-CD163 (0.5 $\mu \mathrm{g} /$ test; cat. no. 17-1639-41). All of the fluorescent-labeled antibodies were obtained from eBioscience, Inc. (San Diego, CA, USA) and incubated with cells for $20 \mathrm{~min}$ at $4^{\circ} \mathrm{C}$ and an Accuri C6 flow cytometer was used (BD Biosciences, Franklin Lakes, NJ, USA).

Statistical analysis. IHC scores were reported as the mean \pm standard error of the mean. Mann-Whitney U test was used to analyze the differences in the expression of B7-H4 between IDC and PC tissues, $\chi^{2}$ test was used to estimate the frequency of B7-H4 expression. The results of ELISA, western blotting and RT-qPCR were analyzed using Student's t-test. All statistical analyses were performed using SPSS 13.0 (SPSS, Inc., Chicago, IL, USA). $\mathrm{P}<0.05$ was considered to indicate a statistically significant difference. 

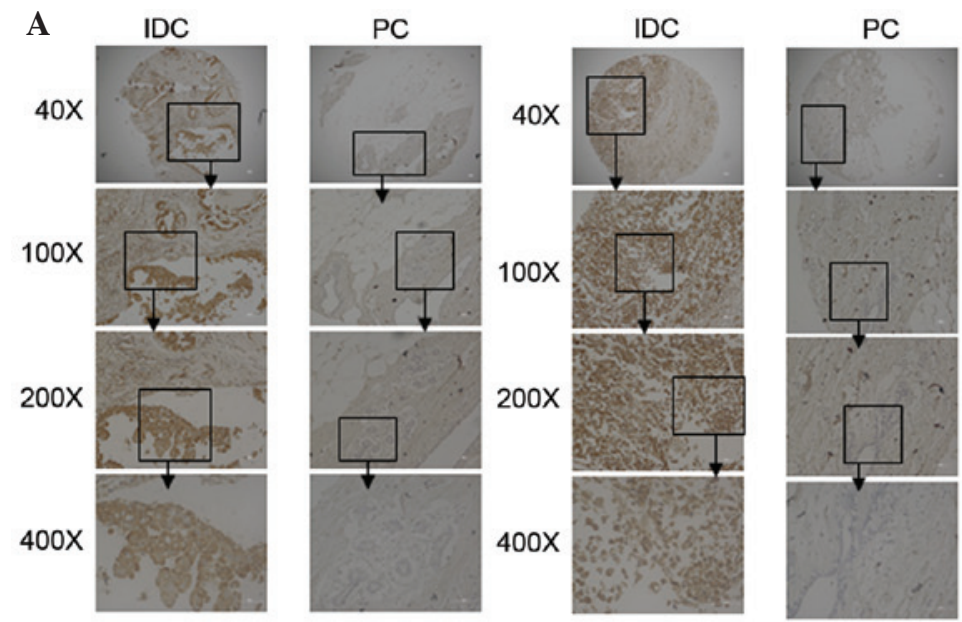

B
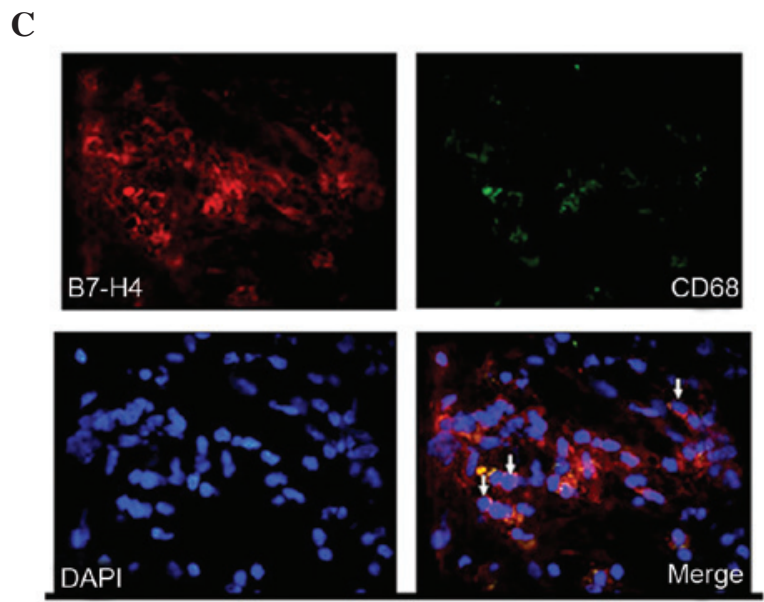

Figure 1. Expression levels of B7-H4 in breast IDC and PC tissues were determined by IHC and immunofluorescence. Samples from 61 patients with a definite pathological diagnosis of IDC were collected in the present study; paired samples of breast IDC and PC tissues were collected from the same patients. (A) Paraffin-embedded sections of breast IDC and PC tissues were prepared for IHC detection. (B) IHC analysis was scored according to the aforementioned methods. Data are presented as the mean \pm standard error of the mean. Mann-Whitney U test was used to analyze the differences in the expression of B7-H4 between IDC and PC tissues (7.10 \pm 0.78 vs. $4.75 \pm 2.08)$. ${ }^{*} \mathrm{P}=0.011$. (C) Co-localization of CD68 and B7-H4 was observed in IDC, the merged images demonstrate the co-localization of the two surface molecules (white arrow) by immunofluorescence (magnification, x400). IDC, infiltrating ductal carcinoma; PC, pericarcinomatous; IHC, immunohistochemsitry; DAPI, 4',6-diamidino-2-phenylindole.

\section{Results}

Breast IDC tissues and tumor-associated macrophages overexpress $\mathrm{B} 7-\mathrm{H} 4$. The expression levels of B7-H4 in breast IDC were characterized in situ. A cohort of 61 patients with IDC was selected (mean age, $53.28 \pm 7.79$ years). Table I summarizes the clinical characteristics of the cohort. Paraffin-embedded sections of IDC and PC samples were stained with a B7-H4 antibody. IHC scores were calculated and used to determine B7-H4 expression. B7-H4 had a higher expression level in the IDC tissues compared with the $\mathrm{PC}$ tissues $(\mathrm{P}=0.011$; Fig. $1 \mathrm{~A}$ and $\mathrm{B}$ and Table I). The positive rate of B7-H4 expression was also significantly higher in the tumor environment compared with the pericarcinomatous tissue as determined by $\chi^{2}$ analysis $(\mathrm{P}<0.01$; data not shown). No significant difference was identified between B7-H4 expression levels and AJCC staging in patients with IDC $(\mathrm{P}>0.05)$. In addition, the expression levels of estrogen receptor (ER), progesterone receptor (PR) and human epidermal growth factor receptor-2 (HER2) were detected in 31 patients. There was a significant difference in B7-H4 expression between IDC and PC tissues in ER-, PR- and HER2-positive and negative patients $(\mathrm{P}<0.05$; Table II). However, when examining IDC tissues alone, no significant difference in B7-H4 expression was identified between ER-, PR- and HER2-positive and negative patients ( $>0.05$; Table II).

Immunofluorescence analysis detected $\mathrm{B} 7-\mathrm{H} 4$ expression in IDC tissue. B7-H4 was shown to be expressed on the surface of macrophages, which were $\mathrm{CD}^{+} 8^{+}$; therefore, it is possible that B7-H4 was not solely expressed on the surface of breast cancer cells but also on macrophages in the breast IDC microenvironment (Fig. 1C).

MO, M1 and M2 cells express various levels of B7-H4. The $\mathrm{CD} 14^{+}$monocytes from PBMC were assessed for purity in the present study, and the proportion of $\mathrm{CD}_{14}{ }^{+}$cells was $>97 \%$ (data not shown). Distinctive surface markers were also highly expressed on macrophages; CD86 was highly expressed on M1 cells and CD163 was highly expressed on M2 cells (Fig. 2A).

Western blot analysis demonstrated that the expression levels of B7-H4 were significantly higher in M2 cells compared 
Table II. Association between clinical characteristics of breast IDC and B7-H4 expression (n=31).

\begin{tabular}{|c|c|c|c|c|}
\hline Variable & Expression & IDC & $\mathrm{PC}$ & P-value \\
\hline ER & $\begin{array}{l}+ \\
-\end{array}$ & $\begin{array}{l}7.05 \pm 0.90 \\
7.08 \pm 0.92\end{array}$ & $\begin{array}{l}5.00 \pm 1.79 \\
5.00 \pm 1.83\end{array}$ & $\begin{array}{l}\mathbf{0 . 0 0 2}^{\mathrm{a}} \\
\mathbf{0 . 0 2 0 ^ { \mathrm { a } }} \\
0.952^{\mathrm{b}}\end{array}$ \\
\hline PR & $\begin{array}{l}+ \\
-\end{array}$ & $\begin{array}{l}6.93 \pm 0.87 \\
7.19 \pm 0.91\end{array}$ & $\begin{array}{l}4.93 \pm 1.70 \\
5.06 \pm 1.94\end{array}$ & $\begin{array}{l}\mathbf{0 . 0 0 5}^{\mathrm{a}} \\
\mathbf{0 . 0 0 6}^{\mathrm{a}} \\
0.446^{\mathrm{b}}\end{array}$ \\
\hline HER 2 & $\begin{array}{l}+ \\
-\end{array}$ & $\begin{array}{l}6.92 \pm 0.94 \\
7.16 \pm 0.89\end{array}$ & $\begin{array}{l}4.75 \pm 2.38 \\
5.16 \pm 1.55\end{array}$ & $\begin{array}{l}\mathbf{0 . 0 4 5 ^ { \mathrm { a } }} \\
\mathbf{0 . 0 0 1}^{\mathrm{a}} \\
0.589^{\mathrm{b}}\end{array}$ \\
\hline
\end{tabular}

Mean IHC score values \pm standard error of the mean were calculated for B7-H4 expression in breast IDC and pericarcinomatous tissues. Statistical differences were calculated using the Mann-Whitney U test. ${ }^{a} \mathrm{P}<0.05 \mathrm{IDC}$ vs. PC tissues. ${ }^{\mathrm{b}} \mathrm{P}>0.05+$ vs. - expression levels of ER, PR or HER2. IDC, infiltrating ductal carcinoma; PC, pericarcinomatous; +, positive; -, negative; ER, estrogen receptor; PR, progesterone receptor; HER2, human epidermal growth factor receptor-2.

A
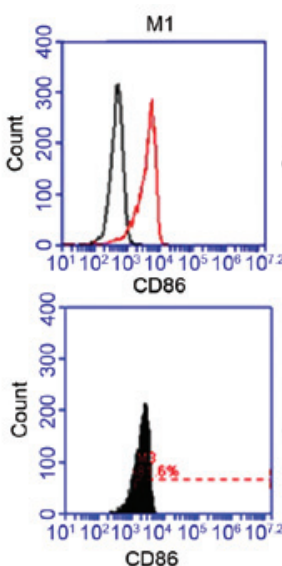

CD86
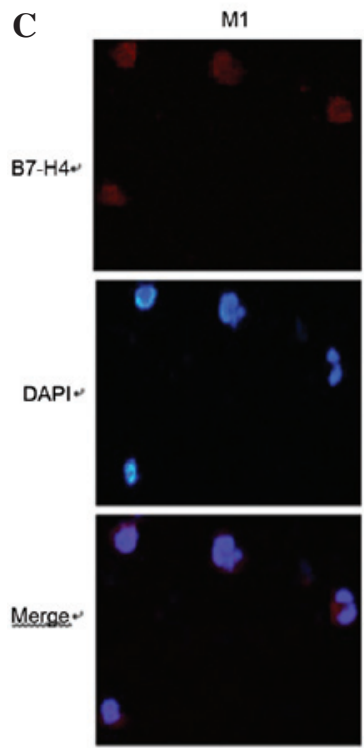

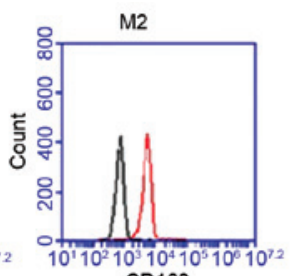

CD163

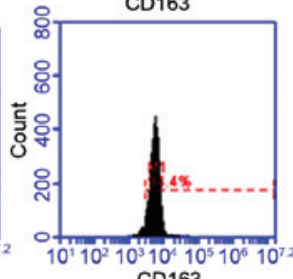

CD163
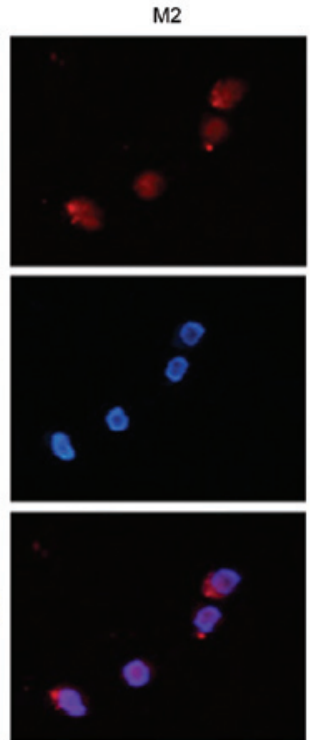

B

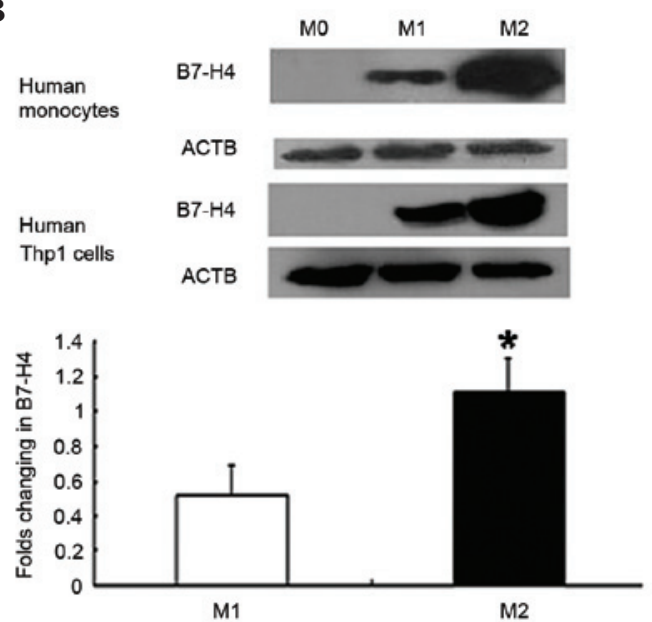

D

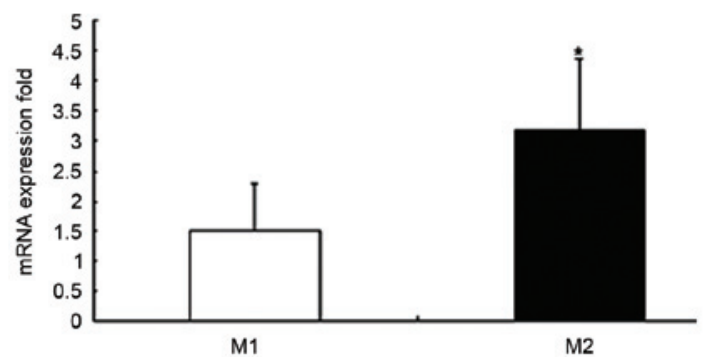

Figure 2. Polarization of macrophages and expression of B7-H4 in various macrophage subtypes. (A) Analysis of the surface markers of M1 and M2 cells using flow cytometry. M1 cells expressed CD86 and M2 cells expressed CD163. (B) Western blot analysis revealed that M2 cells expressed more B7-H4 compared with M1 cells. (C) Macrophages (M1 and M2) were observed to express B7-H4 by immunofluorescence (in red; magnification, x400). (D) M2 cells exhibited higher mRNA expression levels of B7-H4 compared with $\mathrm{M} 1$ cells. ${ }^{*} \mathrm{P}<0.05$. Three independent experiments were performed, data are presented as the mean \pm standard error of the mean. DAPI, 4',6-diamidino-2-phenylindole. 
A
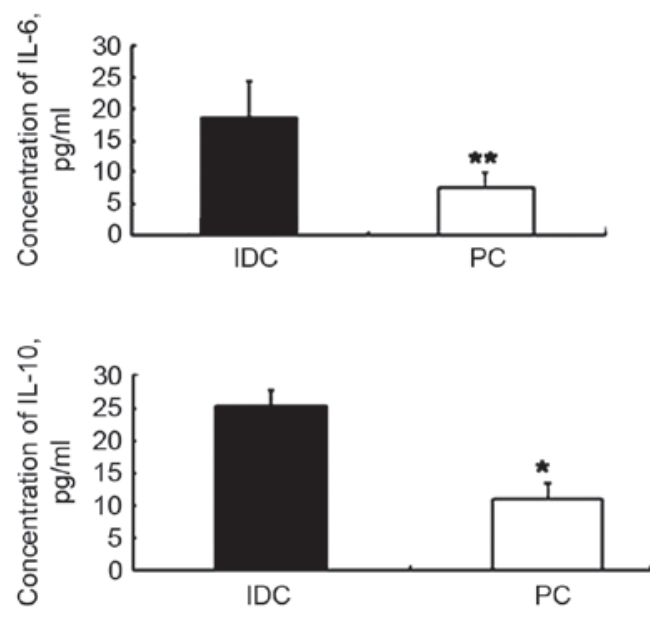

C
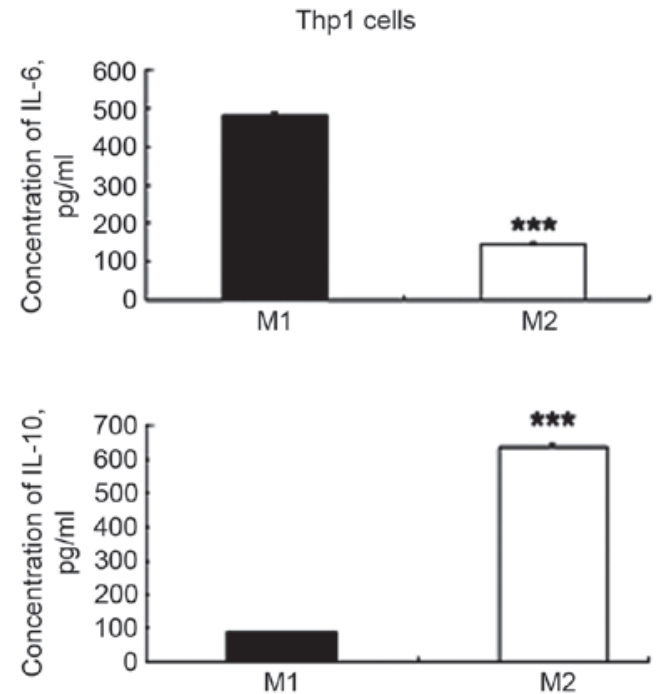
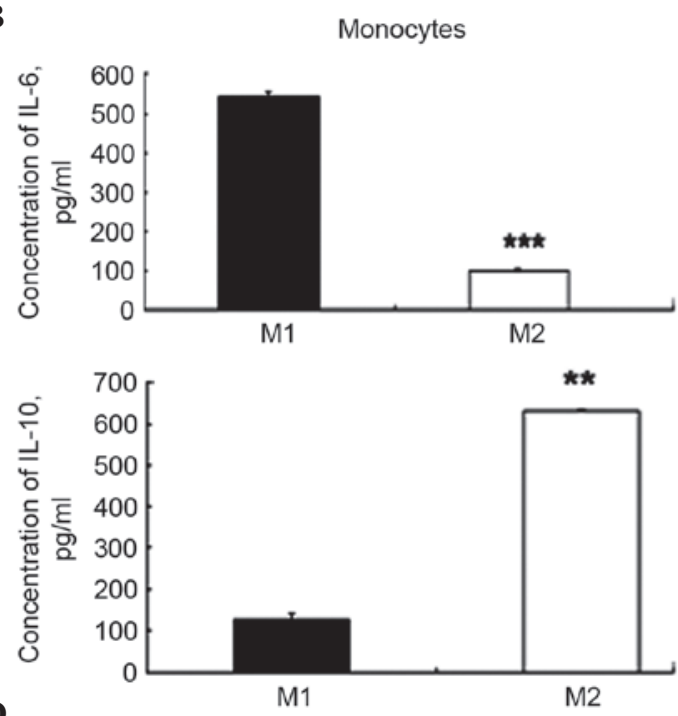

D
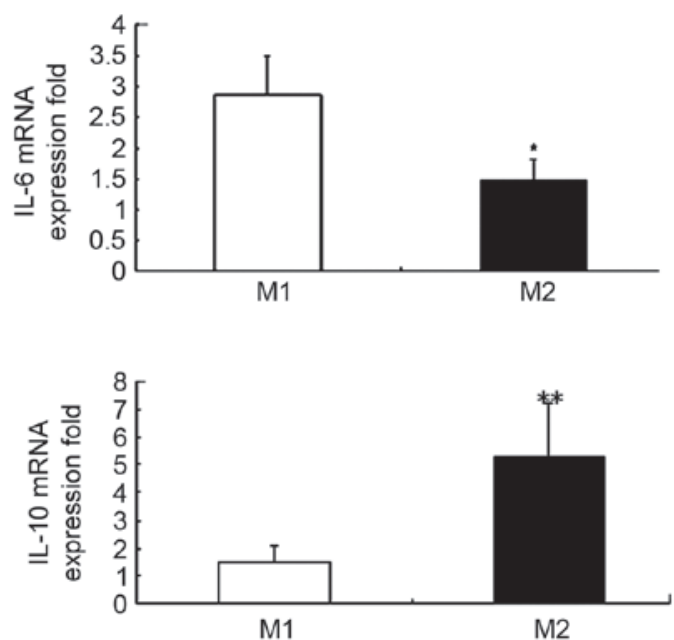

Figure 3. Concentration and expression levels of IL- 6 and -10 in the tumor microenvironment, and M1 and M2 cells. A total of 6 breast IDC and PC samples were used for ELISA. The culture supernatants of M1 and M2 cells were analyzed by ELISA to detect the concentration of IL-6 and -10. (A) Lysate concentrations of IL- 6 and -10 in IDC and PC tissues were observed by ELISA. Supernatant concentrations of IL- 6 and -10 in M1 and M2 cells obtained from (B) CD14 monocytes and (C) Thp1 cells were observed by ELISA. (D) mRNA expression levels of IL-6 and -10 in M1 and M2 cells were determined using reverse transcription-quantitative polymerase chain reaction. ${ }^{*} \mathrm{P}<0.05,{ }^{* * *} \mathrm{P}<0.01,{ }^{* * * *} \mathrm{P}<0.001$. Three independent experiments were performed and data is presented as the mean \pm standard error of the mean. IDC, infiltrating ductal carcinoma; PC, pericarcinomatous; IL, interleukin; ELISA, enzyme-linked immunosorbent assay.

with in M1 cells, in human monocytes and Thp1 cells $(\mathrm{P}<0.05$; Fig. 2B). The verification of the western blot analysis, using a B7-H4 antibody from a different supplier, confirmed this result (data not shown).

Immunofluorescence analysis confirmed that M1 and M2 macrophages expressed B7-H4 (Fig. 2C). B7-H4 expression differed on the surface of M0, M1 and M2 cells, as detected by flow cytometry. RT-qPCR analysis revealed that B7-H4 expression was higher in M2 cells compared with in M1 cells $(\mathrm{P}<0.05$; Fig. 2D).

IL-6 and -10 are highly expressed in IDC tissues. The present study demonstrated that IL-6 and -10 levels were significantly higher in the IDC tissues when compared with the $\mathrm{PC}$ tissues $(\mathrm{P}=0.006$ and $\mathrm{P}=0.031$; Fig. $3 \mathrm{~A})$. There was a significant difference in the average concentrations of IL-6 (18.72 \pm 5.69 vs. $5.80 \pm 2.96 \mathrm{pg} / \mathrm{ml} ; \mathrm{P}=0.006)$ and IL-10
$(27.39 \pm 3.87$ vs. $11.64 \pm 1.77 \mathrm{pg} / \mathrm{ml} ; \mathrm{P}=0.031)$ between IDC and PC tissues. These results suggest that the levels of IL-6 and -10 were three-fold higher in IDC tissues compared with in PC tissues.

High expression levels of IL- 6 in the M1 phenotype and IL-10 in the M2 phenotype. The levels of IL- 6 and -10 in M1 and M2 macrophages obtained from human Thp1 cells and PBMC CD14 ${ }^{+}$monocytes were detected. The ELISA results demonstrated that in M1 cells IL-6 was significantly upregulated compared with in M2 cells (540.54 \pm 13.50 vs. $144.63 \pm 3.24 \mathrm{pg} / \mathrm{ml} ; \mathrm{P}=0.00018$; Fig. 3B). In addition, IL-10 was significantly decreased in M1 cells compared with in M2 cells $(124.61 \pm 14.21$ vs. $628.48 \pm 4.98$ pg/ml, $\mathrm{P}=0.0012$; Fig. 3B). Similar levels were observed in the Thp1 cell line (IL-6, $\mathrm{P}=0.00017$; IL-10, $\mathrm{P}=0.00012$; Fig. 3C). As IL-6 is termed a representative cytokine for the $\mathrm{M} 1$ phenotype in cells and 
IL-10 as one for M2 cells (13), the obtained results fulfilled the expectation of polarization in macrophages.

The RT-qPCR analysis also revealed that the mRNA expression levels of IL-6 were significantly higher in the M1 phenotype when compared with the M2 phenotype $(\mathrm{P}<0.05$; Fig. 3D). The mRNA expression levels of IL-10 were significantly higher in the M2 phenotype compared with the M1 phenotype $(\mathrm{P}<0.01$, Fig. 3D). These results were similar to the results of the ELISA analysis.

\section{Discussion}

Macrophages are important for tumorigenesis due to their secretion of specific cytokines and proteases $(17,18)$. The polarization of macrophages may also affect the progression and metastasis of cancer, including breast cancer (19-22). A previous study functionally classified macrophages into M1 and M2 phenotypes (23). M1 cells, which express CD86 and major histocompatibility complex II, are capable of secreting IL-6 and nitric oxide synthases. However, M2 cells, which express CD163, secrete IL-10 and transforming growth factor- $\beta$ (TGF- $\beta$ ). According to a previous study, macrophages may be stimulated by IL-10, IL- 4 and TGF- $\beta$ in the tumor microenvironment in order to induce the M2 phenotype (13). Due to the characteristics of macrophages in the tumor microenvironment, overexpression of the suppressive co-stimulatory molecule B7-H4 is a possibility.

As a member of the B7 family, B7-H4 has an inhibitory effect on cellular immune responses. Therefore, B7-H4 should be highly expressed in the tumor microenvironment, which is under a state of immune suppression. Previous studies have detected high B7-H4 expression levels in various tumors, including ovarian, lung and breast cancer (24-28). Furthermore, a previous study demonstrated that B7-H4-expressing macrophages were significantly higher in peripheral blood from patients with cancer compared with those from healthy donors (25). Cytokines in peripheral blood may be the induction factors for B7-H4-expressing macrophages in patients with cancer. Therefore, the present study selected human monocytes from peripheral blood and the Thp1 monocyte cell line as targets. The macrophages were stimulated into two different polarization types in vitro. As determined by the detection of surface markers and cytokine secretion, the polarization of macrophages was successful in the present study.

In the tumor microenvironment, M1 cells may limit the development and progression of tumors. Conversely, M2 cells are able to induce tumor promotion $(29,30)$. Due to the inhibitory function of B7-H4, it was hypothesized that M2 macrophages would express higher levels of B7-H4 compared with M1 cells. In the present study, both M1 and M2 human macrophages expressed B7-H4; however, compared with M1 cells, M2 cells exhibited significantly higher levels of B7-H4.

The high expression of B7-H4 in IDC tissues may be due to specific cytokines. Therefore, the present study investigated the levels of IL-6 and -10, and demonstrated that they were higher in IDC tissues compared with PC tissues. This has also been observed in ovarian cancer research, which has revealed that IL-6 and -10 induced B7-H4 expression in macrophages (23). Different concentrations and mRNA expression levels of IL-6 and -10 were observed in the M1 and M2 cells in the present study. Future research will aim to determine the source of IL-6 and -10 in the breast cancer microenvironment, and the association between the expression levels of IL- 6 and -10 and B7-H4 expression in macrophages of different polarization states. IL-6 and -10 may stimulate signal transducer and activator of transcription 3 (Stat3) via the janus kinase/Stat signaling pathway $(31,32)$. Therefore, IL-6 and -10 may be regulated with $\mathrm{B} 7-\mathrm{H} 4$, and the association between $\mathrm{B} 7-\mathrm{H} 4$ and IL-6 or IL-10 signaling regulation should be investigated in future studies.

As a co-inhibitory molecule, B7-H4 has the ability to regulate the immune response in the tumor microenvironment. In addition, B7-H4, as a negative stimulatory molecule, has an inhibitory capacity for activation of the immune response, contrary to the combination of B7-CD28 (33). Therefore, B7-H4 may be a potential target for immunotherapy in various tumors, including breast cancer (34).

In conclusion, the present study indicated that B7-H4 may be overexpressed on the majority of cells in the IDC microenvironment, including macrophages. In vitro experiments revealed that M1 and M2 cells expressed B7-H4. Compared with M1 cells, M2 cells exhibited significantly higher expression levels of B7-H4. In addition, the expression levels of IL-6 and -10 were higher in human breast IDC tissues compared with breast distal PC tissues, and various levels of IL-6 and -10 were observed in the M1 and M2 macrophages.

\section{Acknowledgements}

The present study was supported by the National Natural Science Foundation of China (grant no.81201594), the China Postdoctoral Science Foundation funded project (grant no. 2012M520036) and the Postdoctoral Science Foundation of Heilongjiang Province China (grant no. LRB 2011300).

\section{References}

1. Evans AJ, Pinder SE, Snead DR, Wilson AR, Ellis IO and Elston CW: The detection of ductal carcinoma in situ at mammographic screening enables the diagnosis of small, grade 3 invasive tumours. Br J Cancer 75: 542-544, 1997.

2. Standish LJ, Sweet ES, Novack J, Wenner CA, Bridge C, Nelson A, Martzen M and Torkelson C: Breast cancer and the immune system. J Soc Integr Oncol 6: 158-168, 2008.

3. Watanabe MA, Oda JM, Amarante MK and Cesar Voltarelli J: Regulatory $\mathrm{T}$ cells and breast cancer: Implications for immunopathogenesis. Cancer Metastasis Rev 29: 569-579, 2010.

4. Grieco V, Rondena M, Romussi S, Stefanello D and Finazzi M: Immunohistochemical characterization of the leucocytic infiltrate associated with canine seminomas. J Comp Pathol 130: 278-284, 2004.

5. Carvalho MI, Pires I, Prada J and Queiroga FL: A role for T-lymphocytes in human breast cancer and in canine mammary tumors. Biomed Res Int 2014: 130894, 2014.

6. Gordon S and Taylor PR: Monocyte and macrophage heterogeneity. Nat. Rev Immunol 5: 953-964, 2005.

7. Mantovani A, Sozzani S, Locati M, Allavena P and Sica A: Macrophage polarization: Tumor-associated macrophages as a paradigm for polarized M2 mononuclear phagocytes. Trends Immunol 23: 549-555, 2002.

8. Sica A and Bronte V: Altered macrophage differentiation and immune dysfunction in tumor development. J Clin Invest 117: 1155-1166, 2007.

9. Biswas SK, Sica A and Lewis CE: Plasticity of macrophage function during tumor progression: Regulation by distinct molecular mechanisms. J Immunol 180: 2011-2017, 2008.

10. Qian ZB and Pollard JW: Macrophage diversity enhances tumor progression and metastasis. Cell 141: 39-51, 2010. 
11. Sica GL, Choi IH, Zhu G, Tamada K, Wang SD, Tamura H, Chapoval AI, Flies DB, Bajorath J and Chen L: B7-H4, a molecule of the B7 family, negatively regulates $\mathrm{T}$ cell immunity. Immunity 18: 849-861, 2003.

12. Zang X, Loke P, Kim J, Murphy K, Waitz R and Allison JP. B7x: A widely expressed B7 family member that inhibits $T$ cell activation. Proc Natl Acad Sci USA 100: 10388-10392, 2003.

13. Biswas SK and Mantovani A: Macrophage plasticity and interaction with lymphocyte subsets: Cancer as a paradigm. Nat Immunol 11: 889-896, 2010.

14. Maitra A, Ashfaq R, Gunn CR, Rahman A, Yeo CJ, Sohn TA, Cameron JL, Hruban RH and Wilentz RE: Cyclooxygenase 2 expression in pancreatic adenocarcinoma and pancreatic intraepithelial neoplasia: An immunohistochemical analysis with automated cellular imaging. Am J Clin Pathol 118: 194-201, 2002.

15. Kumar M and Nandi S: Development of a SYBR Green based real-time PCR assay for detection and quantitation of canine parvovirus in faecal samples. J Virol Methods 169: 198-201, 2010.

16. Kabayiza JC, Andersson ME, Welinder-Olsson C, Bergström T, Muhirwa $\mathrm{G}$ and Lindh $\mathrm{M}$ : Comparison of rectal swabs and faeces for real-time PCR detection of enteric agents in Rwandan children with gastroenteritis. BMC Infect Dis 13: 447, 2013.

17. Cheng J, Huo DH, Kuang DM, Yang J, Zheng L and Zhuang SM: Human macrophages promote the motility and invasiveness of osteopontin-knockdown tumor cells. Cancer Res 67: 5141-5147, 2007.

18. Gocheva V, Wang HW, Gadea BB, Shree T, Hunter KE, Garfall AL, Berman T and Joyce JA: IL-4 induces cathepsin protease activity in tumor-associated macrophages to promote cancer growth and invasion. Genes Dev 24: 241-255, 2010.

19. Yang J, Zhang Z, Chen C, Liu Y, Si Q, Chuang TH, Li N, Gomez-Cabrero A, Reisfeld RA, Xiang R and Luo Y: MicroRNA-19a-3p inhibits breast cancer progression and metastasis by inducing macrophage polarization through downregulated expression of Fra-1 proto-oncogene. Oncogene 33: 3014-3023, 2014

20. Jang JY, Lee JK, Jeon YK and Kim CW: Exosome derived from epigallocatechin gallate treated breast cancer cells suppresses tumor growth by inhibiting tumor-associated macrophage infiltration and M2 polarization. BMC Cancer 13: 421, 2013.

21. Oghumu S, Varikuti S, Terrazas C, Kotov D, Nasser MW,Powell CA, Ganju RK and Satoskar AR: CXCR3 deficiency enhances tumor progression by promoting macrophage $\mathrm{M} 2$ polarization in a murine breast cancer model. Immunology 143: 109-119, 2014.

22. Jia X, Yu F, Wang J, Iwanowycz S, Saaoud F, Wang Y, Hu J, Wang Q and Fan D: Emodin suppresses pulmonary metastasis of breast cancer accompanied with decreasedmacrophage recruitment and M2 polarization in the lungs. Breast Cancer Res Treat 148: 291-302, 2014.
23. Mills CD, Kincaid K, Alt JM, Heilman MJ and Hill AM: M-1/M-2 macrophages and the Th1/Th2 paradigm. J Immunol 164: 6166-6173, 2000.

24. Kryczek I, Zou L, Rodriguez P, Zhu G, Wei S, Mottram P, Brumlik M, Cheng P, Curiel T, Myers L, et al: B7-H4 expression identifies a novel suppressive macrophage population in human ovarian carcinoma. J Exp Med 203: 871-881, 2006.

25. Chen C, Zhu YB, Shen Y, Zhu YH, Zhang XG and Huang JA: Increase of circulating B7-H4-expressing CD68+ macrophage correlated with clinical stage of lung carcinomas. J Immunother 35: 354-358, 2012.

26. Salceda S, Tang T, Kmet M, Munteanu A, Ghosh M, Macina R, Liu W, Pilkington G and Papkoff J: The immunomodulatory protein $\mathrm{B} 7-\mathrm{H} 4$ is overexpressed in breast and ovarian cancers and promotes epithelial cell transformation. Exp Cell Res 306: 128-141, 2005

27. Tringler B, Zhuo S, Pilkington G, Torkko KC, Singh M, Lucia MS, Heinz DE, Papkoff J and Shroyer KR: B7-h4 is highly expressed in ductal and lobular breast cancer. Clin Cancer Res 11: 1842-1848, 2005.

28. Mugler KC, Singh M, Tringler B, Torkko KC, Liu W, Papkoff $J$ and Shroyer KR: B7-h4 expression in a range of breast pathology: Correlation with tumor T-cell infiltration. Appl Immunohistochem Mol Morphol 15: 363-370, 2007.

29. Raes G, Brys L, Dahal BK, Brandt J, Grooten J, Brombacher F, Vanham G, Noël W, Bogaert P, Boonefaes T, et al: Macrophage galactose-type C-type lectins as novel markers for alternatively activated macrophages elicited by parasitic infections and allergic airway inflammation. J Leukoc Biol 77: 321-327, 2005.

30. Loke P, Nair MG, Parkinson J, Guiliano D, Blaxter $M$ and Allen JE: IL-4 dependent alternatively-activated macrophages have a distinctive in vivo gene expression phenotype. BMC Immunol 3: 7, 2002.

31. Wang L, Yi T, Kortylewski M, Pardoll DM, Zeng D and Yu H: IL-17 can promote tumor growth through an IL-6-Stat3 signaling pathway. J Exp Med 206: 1457-1464, 2009.

32. Kortylewski M, Kujawski M, Wang T, Wei S, Zhang S, Pilon-Thomas S, Niu G, Kay H, Mulé J, Kerr WG, et al: Inhibiting Stat 3 signaling in the hematopoietic system elicits multicomponent antitumor immunity. Nat Med 11: 1314-1321, 2005.

33. Chen L: Co-inhibitory molecules of the B7-CD28 family in the control of T-cell immunity. Nat Rev Immunol 4: 336-347, 2004.

34. Smith JB, Stashwick C and Powell DJ Jr: B7-H4 as a potential target for immunotherapy for gynecologic cancers: A closer look. Gynecol Oncol 134: 181-189, 2014. 